Review

\title{
Abdominal wall endometriosis (a narrative review)
}

\author{
Mara Carsote ${ }^{1}$, Dana Cristiana Terzea², Ana Valea ${ }^{3}$, Ancuta-Augustina Gheorghisan-Galateanu ${ }^{4 凶}$ \\ 1. Department of Endocrinology, "Carol Davila" University of Medicine and Pharmacy, 050474, Bucharest, Romania; "C.I. Parhon" National Institute of \\ Endocrinology, 011863, Bucharest, Romania \\ 2. Department of Pathology, "C.I. Parhon" National Institute of Endocrinology, 011863, Bucharest, Romania \\ 3. Department of Endocrinology, "Iuliu Hatieganu" University of Medicine and Pharmacy, 400012, Cluj-Napoca, Romania \\ 4. Department of Cellular and Molecular Biology and Histology, “Carol Davila” University of Medicine and Pharmacy, 050474, Bucharest, Romania; “C.I. \\ Parhon" National Institute of Endocrinology, 011863, Bucharest, Romania \\ $\square$ Corresponding author: Ancuta-Augustina Gheorghisan-Galateanu, agheorghisan.a@gmail.com
}

(1) The author(s). This is an open access article distributed under the terms of the Creative Commons Attribution License (https://creativecommons.org/licenses/by/4.0/). See http://ivyspring.com/terms for full terms and conditions.

Received: 2019.07.23; Accepted: 2020.01.27; Published: 2020.02.10

\begin{abstract}
One of the rarest forms of endometriosis is abdominal wall endometriosis (AWE), which includes caesarean scar endometriosis. AWE remains a challenging condition because some issues related to this topic are still under debate. The increasing number of caesarean sections and laparotomies will expect to increase the rate of AWE. The current incidence in obstetrical and gynaecological procedures is still unknown. The disease is probably underestimated. The pathogenic mechanism involves local environment at the implant site including local inflammation and metalloproteinases activation due to local growth factors, estrogen stimulation through estrogen receptors and potential epigenetic changes. However, the underlying mechanisms are not fully explained, and we need more experimental models to understand them. The clinical presentation is heterogeneous; the patient may be seen by a gynaecologist, an endocrinologist, a general surgeon, an imaging specialist, or even an oncologist. No particular constellation of clinical risk factors has been identified, and the histological report is the major diagnostic tool for confirmation. Surgery is the first line of therapy. Further on we need protocols for multidisciplinary investigations and approaches.
\end{abstract}

Key words: endometriosis, abdominal wall endometriosis, caesarean scar endometriosis

\section{Introduction}

Endometriosis, a classic topic of gynaecological endocrinology and a condition that is challenging, is characterized by the presence of endometrial epithelial and stromal cells in non-uterine locations. Endometriosis is typically associated with chronic pain and infertility and affects one in ten women of reproductive age, with different frequencies depending on the site of endometriotic implant [1]. For instance, the most common pelvic locations of endometriotic tissue are the ovary and pelvic peritoneum. Sites of extra-pelvic localization include the gastrointestinal tract, the urinary tract, and the respiratory system $[2,3,4]$. Among these, one of the rarest forms of endometriosis is abdominal wall endometriosis (AWE) or parietal endometriosis, including caesarean scar endometriosis (CSE).
Post-surgical subgroups of endometriosis have increased due to the higher use of caesarean sections worldwide. To date, this particular type of implant is only partially understood, and the diagnosis is often missed and delayed [5,6,7]. The effects of oestrogen exposure after caesarean section and concomitant endometrial seeding during the surgery are enhanced by chronic inflammation, altered immunity, and local growth factors $[1,5,6]$. No particular constellation of clinical risk factors has been identified, and the histological report is the major tool for confirmation, since the preoperative diagnostic rate is low $[7,8]$.

\section{Materials and Methods}

This is a narrative review of the literature based on research using the keywords "endometriosis", 
"abdominal wall endometriosis", and "caesarean scar endometriosis". We mainly included articles published between 2014 and 2019. Due to the rarity of the condition, the highest level of clinical evidence from included papers are observational studies, case series, one case-control study, one prospective cohort study, and some molecular biology-based experimental studies. The aim of this article is to provide an update on AWE from a multimodal and multidisciplinary perspective.

\section{Prevalence}

AWE follows a variety of obstetrical and gynaecological surgeries that are mostly represented by caesarean sections (approximately $85 \%$ by some authors) but also comprise hysterotomy, hysterectomy, and laparoscopic procedures that are performed for non-surgical endometriosis $[3,9,10]$. Sumathy et al. reported concurrent endometriosis in $18.9 \%$ of cases, while others reported no synchronous pelvic lesions $[10,11]$. The mean age at diagnosis is 35 years, and the time from surgery to endometriosis recognition varies from 3 months to 2 decades [11,12].

The reported incidence of CSE is $0.03-0.45 \%$; however, many authors suggest that this low number is due to the rarity of the condition and that the current incidence of AWE (including CSE) cannot be accurately evaluated since consistent epidemiological data are non-existent $[13,14]$. Subcutaneous endometriosis near caesarean scars has been described in only a few isolated cases, including a case of cutaneous endometrial cancer [15]. Recently, a case of scar endometriosis at the level of the uterine cavity was reported [16]. Additionally, 18 cases of trocar port site endometriosis has been reported in the literature [17].

\section{Pathogenic context}

Even though AWE is described by some as the "iatrogenic" subtype of endometriosis, the clear explanation for why some people develop this condition after caesarean section is unclear. In addition to the technical details and precautions themselves, it seems that the pathogenic mechanism is more complex, and endocrine, immune and inflammatory pathways have been considered. While the mechanism is still an enigma, some mechanisms such as metaplasia and cell migration in association with direct seeding have been proposed [18]. Intra-operative implantation is certainly not relevant to non-surgical endometriosis (or "endogenous" endometriosis), and retrograde menstruation (or the Sampson hypothesis) is not involved in postcaesarean section endometriosis, in contrast to pelvic endometriosis $[19,20,21]$. Only a few studies have identified pre-existent pelvic endometriosis $[10,11]$.
The local environment that allows the growth of endometrial cells and stroma includes oestrogen exposure and chronic inflammation [6,19]. Angiogenic growth factor anomalies may be associated with this condition [22].

Genetic and epigenetic changes in endometrial cells are also observed in endometriosis. Genomewide association studies have identified 12 single nucleotide polymorphisms at 10 independent genetic loci that are associated with endometriosis. Two chromosomal areas of significant linkage were observed on 10q26 and 7p13-15 (harbouring genes such as CYP2C19, INHBA, SFRP4 and HOXA10). The identified epigenetic changes comprise methylation and demethylation of DNA and modifications of the histone code $[23,24]$. The genetic/epigenetic theory might explain the heterogeneity of this disease with a hereditary profile, but further studies are needed.

Recently, high expression of PPAR- $\gamma$, a nuclear receptor with anti-inflammatory and neuroprotective roles, has been shown in post-operative lesions, and it has been suggested that PPAR- $\gamma$ could be a pathogenic mechanism of associated pain [25]. In a study focused on "iatrogenic" or "incisional" endometriosis, Lac et al. found that one in four women with this condition had a somatic cancer mutation that may involve two signal transduction pathways, MAPK/RAS or PI3K-Akt-mTor [20].

Non-uterine endometrial cells require metalloproteinases for local remodeling and interaction. These enzymes are activated by local factors, such as TGF $\beta$. Itoh $\mathrm{H}$ et al. showed that stromal endometrial cells of AWE have an abnormal response to TGF $\beta 1$. This may be prevented by progesterone, which does not allow the implant to attach to the local matrix, but it seems that in AWE, there is resistance to progesterone action [26].

Epithelial endometrial glands and stromal cells are positive for oestrogen receptor (ER) expression (Figure 1).

Molecular biology studies of endometriosis have shown the importance of ER as a hallmark of local changes. Endometriotic foci have oestrogen and progesterone receptors that mediate their responsiveness during the menstrual cycle. Methylation defects of genes encoding transcription factors (GATA6, steroidogenic factor-1) and ER $\beta$ cause increased production of oestrogens in the lesion, with secondary inhibition of progesterone receptor. Subsequently, retinol uptake and further metabolization are decreased, causing defects in the endometriotic tissue, with a high level of inflammation and anomalies of prostaglandin production [27]. Moreover, Gou Yet al. showed that the activation of ER $\beta$ in stromal cells is linked to local inflammation 


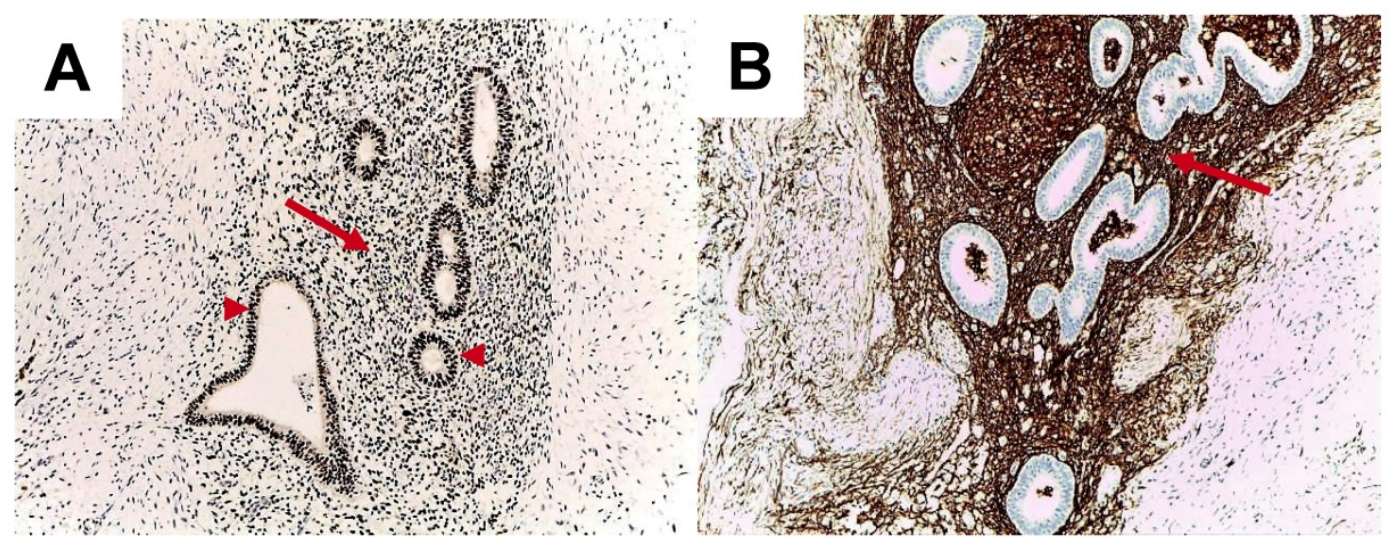

Figure 1. Abdominal wall endometriosis. Immunohistochemistry report. A. High oestrogen receptor positivity in the epithelium of the endometrial glands (arrow heads) and in stroma (arrow). Cell nuclei are stained intensely for estrogen receptors (10x). B. CD10 positivity in the endometrial stroma (arrow) (10x).

because ER induces local CCL2 production through the NF-kB pathway, which triggers local macrophages [28]. Colón-Caraballo et al. demonstrated that the stroma has a tendency for low expression of ERa and progesterone and high expression of ER $\beta$ in the stroma, but the ER $\beta$ : ERa ratio varies with the site of the endometriotic lesion [29].

Overall AWE is developed after surgery only by some females. The mechanisms involve local environment at the implant site including local inflammation and metalloproteinases activation due to local growth factors, estrogen stimulation through estrogen receptors and potential epigenetic changes.

\section{Clinical onset}

Specific symptoms are absent in many cases. Local pain at the caesarean scar/incision site of the abdominal wall during menstruation has been reported to be the most common complaint. Additionally, chronic pain that is unrelated to the menstrual cycle may involve not only the abdominal wall but also the pelvic and lumbar regions [30]. Sometimes, the onset is an acute abdominal emergency [31]. On rare occasions, a patient presents with skin changes; for instance, the patient shows ecchymosis at the level of the abdominal wall during menstruation or hyperpigmentation of a scar (with/ without small local nodules) [13]. A lump may be palpable at the abdominal wall, including on the post-operative scar, with a volume that may vary according to the menstrual cycle [30,32]. Sometimes the lesion is not palpable, and the pain is atypical; thus, the patient is admitted in the general surgery department. Clinical diagnosis is established in $20-50 \%$ of cases, and if additional imaging methods are used, this frequency increases to $70 \%[11,31,32]$. The clinical triangle includes cyclical pain, a lump at or near the level of the scar/abdominal wall and a history of caesarean section or similar gynaecological procedures [3,11]. A study by Zhang et al. showed that the main reason patients present with this condition is abdominal tumour identification (98.5\%), followed by cyclic pain $(86.9 \%)$. Almost $95 \%$ of subjects had only one lump [33]. Regarding the risk factors for AWE, there is not a specific profile. A case-control study by Khan et al. from the Mayo Clinic, in which 2539 females who underwent surgery for endometriosis were enrolled, showed that $1.34 \%$ of the patients had AWE, most frequently (59\%) of CSE type. The accuracy of the diagnosis is increased when independent risk factors, such as the presence of cyclical abdominal pain without dysmenorrhoea and a prior laparotomy, are evident [34]. A study conducted by Andolf et al. showed that the risk for developing endometriosis after caesarean section is $1.8 \%$. [35].

\section{Preoperative investigations}

If AWE is suspected, the most useful assessment tools are ultrasound, computed tomography (CT) and magnetic resonance imaging (MRI) of the abdomen, including the abdominal wall (Figure 2).

MRI is better used in cases with small lesions, while CT provides better results in cases with muscle and subcutaneous layer involvement [36]. Ultrasound remains the best screening method [37]. The mean diameter of the AWE was $4.7 \pm 1.53 \mathrm{~cm}$ in one retrospective observational cohort study [38]. The lesions of AWE have an isoechoic or hyperechoic pattern $(46.7 \%)$, with peripheral vascularization $(61.5 \%)$ on ultrasound and are homogenous and hypervascular on CT scan [39]. MRI is the most commonly used method for evaluating pelvic endometriosis. It is also used for preoperative disease staging [40]. 


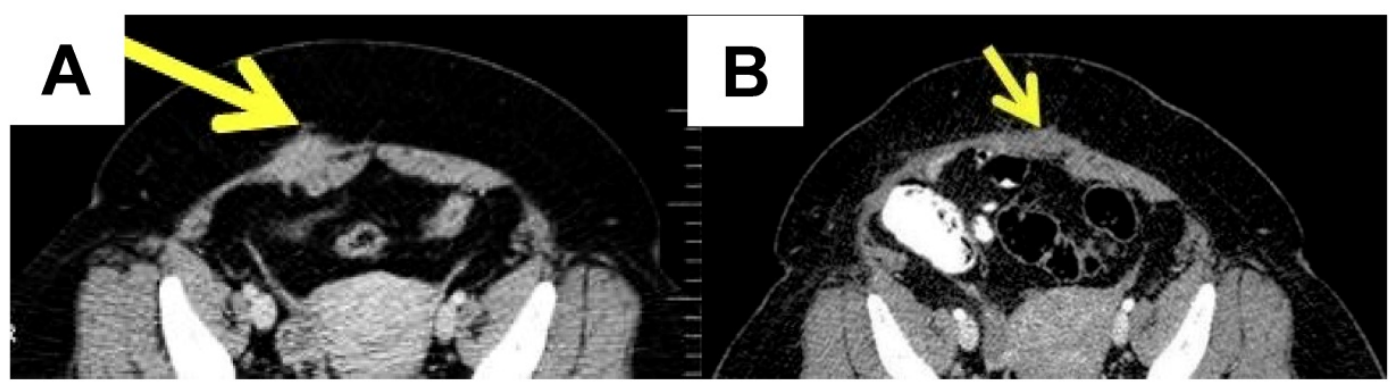

Figure 2. A case of a 44-year-old female diagnosed with abdominal wall endometriosis 14 years after a caesarean section. She had chronic pain unrelated to the menstrual cycle. A. Preoperative aspect: computed tomography showing a poorly defined tumour of $3.9 \mathrm{~cm}$ at the abdominal wall, with a heterogeneous aspect. B. Post-operative aspect by computed tomography.

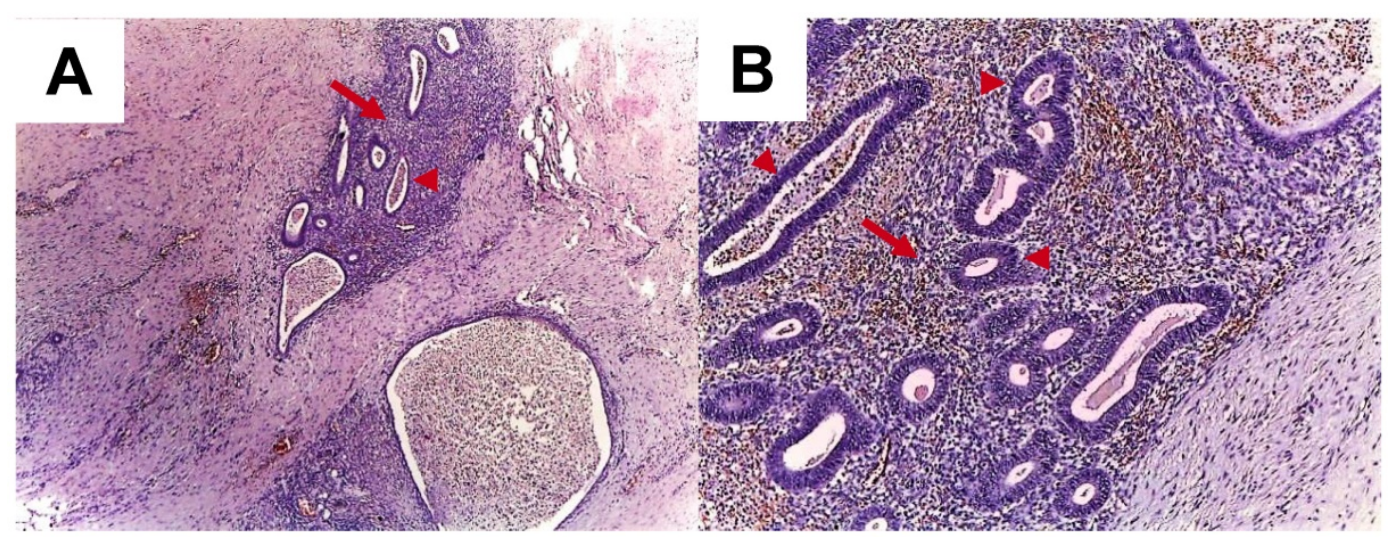

Figure 3. A. Abdominal wall endometriosis. Endometrial glands (arrow heads) and stroma (arrows) in the abdominal wall; HE stain, 4x (A), 10x (B).

Some studies have shown the enhancement of ultrasound accuracy by elastography in the context of abdominal wall infiltration in subjects without excessive fat mass [41]. Transabdominal sonoelastography appears to be particularly useful in lesions of the endometrioma type (but not in patients with a high body mass index) [42]. Positron emission tomography - computed tomography (PET-CT) is less useful because of the low metabolic rate of the cells [38]. Some cases of subcutaneous endometriosis have been evaluated using dermoscopy techniques [43]. Additionally, for superficial lesions, ultrasoundguided fine-needle aspiration has been used depending on the anatomical profile of the lump $[44,45]$. Fine-needle aspiration (FNA) is a simple, non-invasive, easy-to-perform procedure. For instance, in a series of 33 cases, Lopez-Soto et al. used FNA in $72 \%$ of cases [32]. The association between cell block analysis and the cytological report has been shown, and the results of the cytological report have been improved by adding the immunohistochemistry profile based on cell block analysis [46]. FNA is useful for positive diagnosis and for differential diagnosis so it may be the general case' management with a minimal risk of secondary dissemination because the procedure is minimally invasive.

Generally the screening tool remains ultrasound and as a next step MRI or CT is useful.

\section{Pathological report}

Typically, the diagnosis is made after surgery, based on the histological report (Figure 3).

Some tumours are well defined and manifest as endometriomas $[36,47,48]$.

In AWE, endometrial cells are implanted in the rectus abdominis muscle and into the dermis during surgery. Three AWE positions have been described in relation to the rectus abdominis: the superficial implant (above the muscle fascia), intermediate (at the level of the rectus muscle fascia), and the deep position (below the fascia) [49].

The differential diagnosis of AWE includes hernia (inguinal or incisional), abdominal wall tumours of other causes, lipomas, haematomas, granulomas, metastases from distant tumours, and desmoid tumours, among others $[3,11,50,51]$.

\section{Therapy}

AWE requires a multidisciplinary approach. Traditionally, endometriosis is treated by hormonal therapy in addition to pain control drugs and/or surgery, depending on the purpose, namely, pain management and/or achieving fertility [1]. For AWE and CSE cases, surgery is the only curative therapy, and the removal of the lump also causes chronic pain to disappear [12,48]. Preoperative radioisotope 
injection has recently been used to clearly identify small lesions during resection but there are limited data [52]. A wide incision for endometriotic nodules is recommended due to the risk of recurrence described in $5-9 \%$ of cases $[16,32]$. Sclerotherapy with ultrasound guided ethanol injection into the lesion of scar endometriosis has been reported to be effective in isolated cases to prevent abdominal wall defects after wide excision [53]. Recently, as an alternative to surgery, some authors have suggested, high-intensity focused ultrasound ablation (HIFUA), which has a recurrence rate of $3.9 \%$ [54,55]. Lee JS et al. showed that the rate of side effects, such as blood loss and parietal defects, is lower when HIFUA is used against AWE [56]. Combined oral contraceptives, progestogens and hormone suppression therapy with gonadotropin-releasing hormone $(\mathrm{GnRH})$ analogues are useful for patients who refuse surgery or for post-operative management to reduce the risk of recurrence and delay new growth. Additionally, previous hormonal treatment may be an option for larger tumours and may reduce their sizes before surgery. However, the clinical improvement observed for endometriotic implants at other sites has not been observed for AWE [57]. The main therapeutically approach is the surgical remove.

\section{Malignancy risk}

Endometriosis of any site has an associated malignancy risk of $1 \%$. Eighty percent of malignancy cases are related to endometriosis located at the ovary, and $20 \%$ of these cases are related to extra-gonadal locations (including the abdominal wall) [58]. Genetic anomalies, such as loss of heterozygosity or PTEN, ARID1A or p53 mutations, have been implicated [59]. Local production of reactive oxygen species and prolonged oestrogen exposure may increase the risk of malignant transformation [60].

Malignant evolution is suspected in AWE cases with rapid growth of the endometriotic implant [18]. In 2017, a PRISMA systematic review was published in relation to the malignancy risk of endometriosis following obstetrical surgery. This systematic review based on prior reviews and case reports included 47 cases diagnosed with AWE-related cancer between 1980 and 2016. A total of $87 \%$ of patients had a previous caesarean section, while $13 \%$ had other types of gynaecological procedures. The median period of time from surgery to cancer diagnosis was 19 years [9]. Previous data suggested an interval of up to 39 years [61,62]. The median survival time was 42 months, with a poor prognosis for clear cell adenocarcinoma followed by endometrioid adenocarcinoma $[63,64]$. A prior review indicated a percentage of $44 \%$ mortality within the first few months after diagnosis [61]. The treatment for endometriosis-associated malignant transformation in an abdominal surgical scar is extensive surgery and adjuvant chemotherapy and/or radiotherapy.

\section{Conclusion}

AWE represents a dynamic, yet incompletely known, multidisciplinary topic. The incidence is increasing due to the increasing number of obstetrical and gynaecological procedures. The clinical aspects range from a lump to local pain at the abdominal wall or caesarean scar. Imaging techniques like ultrasound and magnetic resonance may help but the definitive diagnosis is based on a post-operative histological report. Surgical removal of the implant currently represents the best management. The questions that still do not have a clear answer are: the true prevalence in the female population; the risk of recurrence after an initial surgical approach; the rate of malignant transformation; the underlying seeding mechanisms and pathways of cancer related. Moreover, standard protocols are needed.

\section{Abbreviations}

AWE: abdominal wall endometriosis; ARI1A: AT-rich interactive domain-containing protein 1A; CCL2: chemokine (C-C motif) ligand 2; CYP2C19: cytochrome P450 2C19; CSE: caesarean scar endometriosis; CT: computed tomography; DNA: deoxyribonucleic acid; ER: oestrogen receptor; FNA: fine-needle aspiration; GABA6: gamma-aminobutyric acid 6; GnRH: gonadotropin-releasing hormone; HIFUA: high-intensity focused ultrasound ablation; HOXA10: homeobox protein Hox-A10; INHBA: inhibin, beta A; MRI: magnetic resonance imaging; MAPK: mitogen-activated protein kinases; NF-kB: nuclear factor kappa-light-chain-enhancer of activated B cells; PET-CT: positron emission tomography - computed tomography; PPAR- $\gamma$ : peroxisome proliferator-activated receptor gamma; PI3K-Akt-mTor: phosphatidylinositol-3-kinase (PI3K) /Akt and the mammalian target of rapamycin (mTOR); PTEN: phosphatase and tensin homolog; P53: tumor protein p53; SFRP4: secreted frizzled-related protein 4 ; TGF $\beta$ : transforming growth factor $\beta$.

\section{Acknowledgements}

\section{Authorship}

MC was involved in the design of the study, data collection, literature review, and manuscript conception. DCT was involved in performing histological and immunohistochemical staining and examinations, performing pathology-based diagnoses and obtaining 
microscopy photos. AV was involved in the acquisition and analysis of data, the literature search and the drafting of the manuscript. AAGG was involved in the manuscript's conception, microscopic image analysis, microscopic image processing into the final form for publication, and critical revision of the manuscript for important intellectual content. All authors have read and approved the final manuscript.

\section{Ethics approval and consent to participate}

This study adhered to the tenets of the 1964 Declaration of Helsinki.

\section{Competing Interests}

The authors have declared that no competing interest exists.

\section{References}

1. Zondervan KT, Becker CM, Koga K, et al. Endometriosis. Nat Rev Dis Primers. 2018; 4: 9 .

2. Lainas P, Dammaro C, Rodda GA, et al. Appendiceal endometriosis invading the sigmoid colon: a rare entity. Int J Colorectal Dis. 2019; 34: 1147-50.

3. Alsinan TA, AlDahleh LA, Alreefi HAA, et al. Endometriosis of the Urinary Bladder Causing a Right Hydronephrosis: A Case Report. Am J Case Rep. 2019; 14: 1360-3.

4. Tong SS, Yin XY, Hu SS, et al. Case report of pulmonary endometriosis and review of the literature. J Int Med Res. 2019; 47: 1766-70.

5. Grigore M, Socolov D, Pavaleanu I, et al. Abdominal wall endometriosis: an update in clinical, imagistic features, and management options. Med Ultrason. 2017; 19: 430-7.

6. Morales Martínez C, Tejuca Somoano S. Abdominal wall endometriosis. Am J Obstet Gynecol. 2017; 217: 701-2.

7. Koninckx PR, Ussia A, Wattiez A, et al. Risk Factors, Clinical Presentation, and Outcomes for Abdominal Wall Endometriosis. J Minim Invasive Gynecol. 2018; 25: 342-3.

8. Ince C, Wagner A, Rajakumar C. Abdominal Wall Endometriosis. J Obstet Gynaecol Can. 2018; 40: 859.

9. Mihailovici A, Rottenstreich M, Kovel S, et al. Endometriosis-associated malignant transformation in abdominal surgical scar: A PRISMA - compliant systematic review. Medicine (Baltimore). 2017; 96: e9136. doi: 10.1097/ MD.0000000000009136.

10. Sumathy S, Mangalakanthi J, Purushothaman K, et al. Symptomatology and Surgical Perspective of Scar Endometriosis: A Case Series of 16 Women. J Obstet Gynaecol India. 2017; 67: 218-23.

11. Tatli F, Gozeneli $\mathrm{O}$, Uyanikoglu $\mathrm{H}$, et al. The clinical characteristics and surgical approach of scar endometriosis: A case series of 14 women. Bosn J Basic Med Sci. 2018; 18: 275-8.

12. Pas K, Joanna SM, Renata R, et al. Prospective study concerning 71 cases of caesarean scar endometriosis (CSE). J Obstet Gynaecol. 2017; 37: 775-8.

13. Alnafisah F, Dawa SK, Alalfy S. Skin Endometriosis at the Caesarean Section Scar: A Case Report and Review of the Literature. Cureus. 2018; 10: e2063. doi: 10.7759/cureus.2063.

14. Tajima $S$, Bito $T$, Ikeda $T$, et al. Cutaneous endometrial cancer arising from hetero-topic endometriosis in an abdominal caesarean section scar. J Eur Acad Dermatol Venereol. 2016; 30: 683-5.

15. D'Agostino C, Surico D, Monga G, et al. Pregnancy-related decidualization of sub-cutaneous endometriosis occurring in a post-caesarean section scar: Case study and review of the literature. Pathol Res Pract. 2019; 215: 828-31.

16. Yin $\mathrm{W}, \mathrm{Zhang} \mathrm{J}, \mathrm{Xu} \mathrm{L}$, et al. Intrauterine endometrial cyst after low uterine incision: A case report with literature review. Medicine (Baltimore). 2018; 97: e0376. doi: 10.1097/ MD. 0000000000010376.

17. Akbarzadeh-Jahromi M, Motavas M, Fazelzadeh A. Recurrent abdominal wall endometriosis at the trocar site of laparoscopy: A rare case. Int J Reprod Biomed (Yazd). 2018; 16: 653-6.

18. Vellido-Cotelo R, Muñoz-González JL, Oliver-Pérez MR, et al. Endometriosis node in gynaecologic scars: a study of 17 patients and the diagnostic considerations in clinical experience in tertiary care center. BMC Womens Health. 2015; 15: 13. doi: 10.1186/ s12905-015-0170-9.

19. Yildırım D, Tatar C, Doğan O, et al. Post-cesarean scar endometriosis. Turk J Obstet Gynecol. 2018; 15: 33-8.

20. Lac V, Verhoef L, Aguirre-Hernandez R, et al. Iatrogenic endometriosis harbors somatic cancer-driver mutations. Hum Reprod. 2019; 34: 69-78.

21. Davis AC, Goldberg JM. Extrapelvic Endometriosis. Semin Reprod Med. 2017; 35: 98-101.
22. Malutan A, Drugan T, Georgescu C, et al. Vascular Endothelial Growth Factor Serum Levels in Women with Advanced Endometriosis. Acta Endo (Buc) 2016; 12: 7-13.

23. Borghese B, Zondervan KT, Abrao MS, Chapron C, Vaiman D. Recent insights on the genetics and epigenetics of endometriosis. Clin Genet. 2017; 91: 254-64.

24. Koninckx PR, Ussia A, Adamyan L, et al. Pathogenesis of endometriosis: the genetic/ epigenetic theory. Fertil Steril. 2019; 111: 327-40.

25. Harzif AK, Silvia M, Mariana A, et al. Extrapelvic endometriosis in abdominal wall scar and PPAR gamma expression: A case report. Int J Surg Case Rep. 2018; 53: 66-69.

26. Itoh H, Mogami H, Bou Nemer L, et al. Endometrial stromal cell attachment and matrix homeostasis in abdominal wall endometriomas. Hum Reprod. 2018; 33: 280-91.

27. Bulun SE, Monsivais D, Kakinuma $\mathrm{T}$, et al. Molecular biology of endometriosis: from aromatase to genomic abnormalities. Semin Reprod Med. 2015; 33: 220-4.

28. Gou Y, Li X, Li P, et al. Estrogen receptor $\beta$ upregulates CCL2 via NF-kB signaling in endometriotic stromal cells and recruits macrophages to promote the pathogenesis of endometriosis. Hum Reprod. 2019; 34: 646-58.

29. Colón-Caraballo M, García M, Mendoza A, et al. Human Endometriosis Tissue Microarray Reveals Site-specific Expression of Estrogen Receptors, Progesterone Receptor, and Ki67. Appl Immunohistochem Mol Morphol. 2019; 27: 491-500.

30. Sedhain N, Dangal G, Karki A, et al. Caesarean Scar Endometriosis. J Nepal Health Res Counc. 2018; 15: 292-4.

31. Roi DP, Schamroth JL, Khalid L, et al. Scar endometriosis: a mimic of acute abdominal emergencies. BJR Case Rep. 2017; 3: 20170019. doi: 10.1259 /bjrcr.20170019.

32. Lopez-Soto A, Sanchez-Zapata MI, Martinez-Cendan JP, et al. Cutaneous endometriosis: Presentation of 33 cases and literature review. Eur J Obstet Gynecol Reprod Biol. 2018; 221: 58-63.

33. Zhang P, Sun $Y$, Zhang C, et al. Cesarean scar endometriosis: presentation of 198 cases and literature review. BMC Womens Health. 2019; 19: 14. doi: 10.1186/s12905-019-0711-8.

34. Khan Z, Zanfagnin V, El-Nashar SA, et al. Risk Factors, Clinical Presentation, and Outcomes for Abdominal Wall Endometriosis. J Minim Invasive Gynecol. 2017; 24: 478-84.

35. Andolf $\mathrm{E}$, Thorsell $\mathrm{M}$, Källén $\mathrm{K}$. Caesarean section and risk for endometriosis: a prospective cohort study of Swedish registries. BJOG. 2013; 120: 1061-5.

36. Menon M, T A S, P N C, et al. Skin to serosa: scar endometrioma. J Clin Diagn Res. 2014; 8: OD04-5. doi:10.7860/JCDR/2014/8894.4985.

37. Oh EM, Lee WS, Kang JM, et al. A Surgeon's Perspective of Abdominal Wall Endometriosis at a Caesarean Section Incision: Nine Cases in a Single Institution. Surg Res Pract. 2014; 2014: 765372. doi: 10.1155/2014/765372.

38. Hocaoglu $\mathrm{M}$, Turgut $\mathrm{A}$, Ozdamar $\mathrm{O}$, et al. Abdominal wall endometriosis in patients with a history of cesarian section. Ann Ital Chir. 2018; 89: 425-30.

39. Jaramillo-Cardoso A, Balcacer P, Garces-Descovich A, et al. Multimodality imaging and clinicopathologic assessment of abdominal wall endometriosis: knocking down the enigma. Abdom Radiol (NY). 2018; doi:10.1007/s00261-018-1666-1.

40. Foti PV, Farina R, Palmucci S, et al. Endometriosis: clinical features, MR imaging findings and pathologic correlation. Insights Imaging. 2018; 9: 149-72.

41. Wozniak S, Czuczwar P, Szkodziak P, et al. Elastography Improves the Accuracy of Ultrasound in the Preoperative Assessment of abdominal wall endometriosis. Ultraschall Med. 2015; 36: 623-9.

42. Fawzy M, Amer T. Efficacy of transabdominal sonoelastography in the diagnosis of caesarean section scar endometrioma: A pilot study. J Obstet Gynaecol. 2015; 35: 832-4.

43. Tognetti L, Cinotti E, Tonini G, et al. New findings in non-invasive imaging of cutaneous endometriosis: Dermoscopy, high-frequency ultrasound and reflectance confocal microscopy. Skin Res Technol. 2018; 24: 309-12.

44. Pachori G, Sharma R, Sunaria RK, et al. Scar endometriosis: Diagnosis by fine needle aspiration. J Cytol. 2015; 32: 65-7.

45. Ail DA, Joshi AR, Manzoor I, et al. Fine-needle Aspiration Cytology of Abdominal Wall Endometriosis: A Meaningful Adjunct to Diagnosis. Oman Med J. 2018; 33: 72-5.

46. Dash S, Panda S, Rout N, et al. Role of fine needle aspiration cytology and cell block in diagnosis of scar endometriosis: A case report. J Cytol. 2015; 32: 71-3.

47. Kocher M, Hardie A, Schaefer A, et al. Cesarean-Section Scar Endometrioma: A Case Report and Review of the Literature. J Radiol Case Rep. 2017; 11: 16-26.

48. Ozturk A, Kaya C, Bozkurtoglu H, et al. Scar Endometrioma: An Uncommon Yet Easily Treated Condition. J Reprod Med. 2016; 61: 249-53.

49. Goker A, Sarsmaz K, Pekindil G, et al. Rectus abdominis muscle endometriosis. J Coll Physicians Surg Pak. 2014; 24: 944-6.

50. Patil NJ, Kumar V, Gupta A. Scar endometriosis-a sequel of caesarean section. J Clin Diagn Res. 2014; 8: FD09-FD10.

51. Nambiar R, Anoop TM, Mony RP. Abdominal Wall Endometriosis Mimicking Metastases. Indian J Surg Oncol. 2018; 9: 278-9.

52. Vitral GSF, Salgado HC, Rangel JMC. Use of radioguided surgery in abdominal wall endometriosis: An innovative approach. World J Nucl Med. 2018; 17: 204-6.

53. Bozkurt M, Cil AS, Bozkurt DK. Intramuscular abdominal wall endometriosis treated by ultrasound-guided ethanol injection. Clin Med Res. 2014; 12: 160-5.

54. Xiao-Ying Z, Hua D, Jin-Juan W, et al. Clinical analysis of high-intensity focussed ultrasound ablation for abdominal wall endometriosis: a 4-year 
experience at a specialty gynecological institution. Int J Hyperthermia. 2019; 36: 87-94.

55. Zhao L, Deng $\mathrm{Y}$, Wei Q, et al. Comparison of ultrasound-guided high-intensity focused ultrasound ablation and surgery for abdominal wall endometriosis. Int J Hyperthermia. 2018; 35: 528-33.

56. Lee JS, Kim YJ, Hong GY, et al. Abdominal wall endometriosis treatment by ultrasound-guided high-intensity focused ultrasound ablation: a case report. Gynecol Endocrinol. 2019; 35: 109-11.

57. Touleimat S, Darwish B, Vassilieff M, et al. Abdominal wall endometriosis following cesarean section: a study of the growth rate of parietal endometriosis implants. Minerva Ginecol. 2017; 69: 440-6.

58. Ferrandina G, Palluzzi E, Fanfani F, et al. Endometriosis-associated clear cell carcinoma arising in caesarean section scar: a case report and review of the literature. World J Surg Oncol. 2016; 14: 300.

59. Krawczyk N, Banys-Paluchowski M, Schmidt D, et al. Endometriosisassociated Malignancy. Geburtshilfe Frauenheilkd. 2016; 76: 176-81.

60. Kajiyama H, Suzuki S, Yoshihara M, et al. Endometriosis and cancer. Free Radic Biol Med. 2019; 133: 186-92.

61. Taburiaux L, Pluchino N, Petignat P, et al. Endometriosis-Associated Abdominal Wall Cancer: A Poor Prognosis? Int J Gynecol Cancer. 2015; 25: 1633-8.

62. Dobrosz Z, Paleń P, Stojko R, et al. Clear cell carcinoma derived from an endometriosis focus in a scar after a caesarean section- a case report and literature review. Ginekol Pol. 2014; 85: 792-5.

63. Graur F, Mois E, Elisei R, et al. Malignant endometriosis of the abdominal wall. Ann Ital Chir. 2017; 6: S2239253X17026895.

64. Lai YL, Hsu HC, Kuo KT, et al. Clear Cell Carcinoma of the Abdominal Wall as a Rare Complication of General Obstetric and Gynecologic Surgeries: 15 Years of Experience at a Large Academic Institution. Int J Environ Res Public Health. 2019; 16: 552. 